Relations industrielles

Industrial Relations

\title{
Le changement du lieu de travail - étude d'un cas de reclassement, par Grégoire Tremblay avec la collaboration de Isabel Romao, Montréal, direction générale de la recherche, ministère du Travail et de la Main-d'oeuvre, 1973, 160 pp.
}

\section{A. Legault}

Volume 28, numéro 4, 1973

URI : https://id.erudit.org/iderudit/028469ar

DOI : https://doi.org/10.7202/028469ar

Aller au sommaire du numéro

Éditeur(s)

Département des relations industrielles de l'Université Laval

ISSN

0034-379X (imprimé)

1703-8138 (numérique)

Découvrir la revue

Citer ce compte rendu

Legault, I. A. (1973). Compte rendu de [Le changement du lieu de travail - étude d'un cas de reclassement, par Grégoire Tremblay avec la collaboration de Isabel Romao, Montréal, direction générale de la recherche, ministère du Travail et de la Main-d'oeuvre, 1973, 160 pp.] Relations industrielles / Industrial Relations, 28(4), 899-899. https://doi.org/10.7202/028469ar

Tous droits réservés @ Département des relations industrielles de l'Université Laval, 1973
Ce document est protégé par la loi sur le droit d'auteur. L'utilisation des services d'Érudit (y compris la reproduction) est assujettie à sa politique d'utilisation que vous pouvez consulter en ligne.

https://apropos.erudit.org/fr/usagers/politique-dutilisation/ 
méthodologie utilisée dans des travaux de recherche en administration. Une seconde partie couvre des thèmes classiques tels que le processus administratif, la prise de décision, les politiques, la planification, la délégation, l'envergure de la supervision. La dernière s'inspire beaucoup plus de l'approche des sciences du comportement en retenant les idées les plus récentes sur la motivation, le leadership, la formation des cadres et le développement des organisations.

La valeur d'un recueil de ce genre repose pour une bonne part sur l'agencement du matériel; mais surtout sur la qualité des articles retenus. Sur ce dernier point, le volume est excellent. La majorité des articles figurent parmi les meilleurs que je connaisse sur tous les thèmes. A titre d'exemple, le travail de R.J. House et L.A. Wigdor présente une synthèse et une critique des recherches effectuées sur la validité de la théorie de Herzberg.

Evidemment, ceux qui peuvent lire la plupart des revues spécialisées en sciences sociales et en management ne verront rien d'original dans ce recueil. Mais, pour les étudiants qui veulent économiser sur les frais de la photocopie et pour les praticiens qui veulent compléter leurs connaissances dans le domaine des sciences du comportement et de l'administration, c'est un ouvrage que je n'hésite pas à recommander.

\section{Université Laval}

\section{Laurent BELANGER}

\section{Le changement du lieu de travail - étude} d'un cas de reclassement, par Grégoire Tremblay avec la collaboration de Isabel Romao, Montréal, direction générale de la recherche, ministère du Travail et de la Main-d'œuvre, 1973, $160 \mathrm{pp}$.

Comme son titre l'indique, il s'agit d'une étude portant sur l'adaptation des travailleurs sous l'aspect particulier de la mobilité géographique.

Une première partie identifie et discute certains facteurs qui influent sur la mobilité géographique des travailleurs alors que la deuxième porte sur l'étude d'un cas de reclassement dans lequel la mobilité géographique des travailleurs fut envisagée comme l'une des mesures possibles d'adaptation de la main-d'oeuvre susceptible d'être appliquée par suite da déménagement de l'établissement de leur employeur dans une autre localité.

Dans cet ouvrage les auteurs mettent en lumière un certain nombre de considérations ayant rapport au facteur humain dont on fait ressortir toute l'importance lorsque le déménagement d'un travailleur d'une localité à une autre localité doit être envisagé parmi les mesures d'adaptation applicables dans une opération de reclassement.

Bien que la mobilité géographique ne soit qu'un seul parmi nombre d'autres éléments à considérer dans l'opération complète de reclassement, la deuxième partie de l'étude sous-tend une évaluation globale de l'opération de reclassemient poursuivie dans le cas témoin étudié. Vue sous cet angle l'étude nous paraît incomplète. Ainsi, il eut été important de connaître le point de vue de l'employeur sur l'ensemble de l'opération et la participation qu'il entendait y contribuer en vue de faciliter l'adaptation de ses employés. Il eut été intéressant de savoir aussi quelles furent d'après lui les facteurs inhibitifs d'un plus grand succès de l'adaptation de ses employés.

La présence syndicale, s'il y avait, n'est pas apparente à travers les interviews. On aurait aimé, de ce point de vue, connaître la réaction syndicale sur le résultat des interviews et avoir une explication sur la carence apparente de la participation syndicale et même de la participation des employés eux-mêmes. I1 y aurait également eut lieu d'examiner toutes les démarches et recommandations issues du comité conjoint de reclassement en rapport avec la mobilité vue cependant à travers l'ensemble des dispositions d'adaptation et selon l'interprétation donnée par l'employeur et par le syndicat.

Bref, malgré ses lacunes cet essai mérite cependant d'être lu de tous ceux qui oeuvrent dans la recherche se rapportant aux problèmes d'adaptation de la main-d'oeuvre ou qui se préoccupent de leur solution.

\section{A. LEGAULT}

Ministère des la Main-d'œuvre et de l'Immigration, Ottawa 